\title{
Risk Factors for Mortality in Hemodialysis Patients: Two-Year Follow-Up Study
}

\author{
Maria do Sameiro-Faria, , ${ }^{1,2}$ Sandra Ribeiro, ${ }^{3,4}$ Elísio Costa, ${ }^{3,4}$ Denisa Mendonça, ${ }^{1,5}$ \\ Laetitia Teixeira, ${ }^{1}$ Petronila Rocha-Pereira, ${ }^{4,6}$ João Fernandes, ${ }^{4,7}$ Henrique Nascimento, ${ }^{3,4}$ \\ Michaela Kohlova, ${ }^{6}$ Flávio Reis, ${ }^{6}$ Leonilde Amado, ${ }^{2}$ Elsa Bronze-da-Rocha, ${ }^{3,4}$ \\ Vasco Miranda, ${ }^{2}$ Alexandre Quintanilha, ${ }^{1,4}$ Luís Belo, ${ }^{3,4}$ and Alice Santos-Silva ${ }^{3,4,8}$ \\ ${ }^{1}$ Instituto de Ciências Biomédicas Abel Salazar, Universidade do Porto, Porto, Portugal \\ ${ }^{2}$ Nephrocare Portugal, SA-Nephrocare Maia, Maia, Portugal \\ ${ }^{3}$ Laboratório de Bioquímica, Departamento de Ciências Biológicas, Faculdade Farmácia, Universidade do Porto, Porto, Portugal \\ ${ }^{4}$ Instituto de Biologia Molecular e Celular (IBMC), Universidade do Porto, Porto, Portugal \\ ${ }^{5}$ Instituto de Saúde Pública, Universidade do Porto, Porto, Portugal \\ ${ }^{6}$ Centro Investigação Ciências Saúde, Universidade Beira Interior, Covilhã, Portugal \\ ${ }^{7}$ IBILI, Faculdade de Medicina, Universidade de Coimbra, Coimbra, Portugal \\ ${ }^{8}$ Serviço de Bioquímica, Departamento de Ciências Biológicas, Faculdade de Farmácia, Universidade do Porto, \\ Rua Jorge Viterbo Ferreira 228, 4050-313 Porto, Portugal
}

Correspondence should be addressed to Alice Santos-Silva; assilva@ff.up.pt

Received 24 January 2013; Accepted 2 May 2013

Academic Editor: Sudhir Srivastava

Copyright (C) 2013 Maria do Sameiro-Faria et al. This is an open access article distributed under the Creative Commons Attribution License, which permits unrestricted use, distribution, and reproduction in any medium, provided the original work is properly cited.

Background. End-stage renal disease (ESRD) patients under hemodialysis (HD) have high mortality rate. Inflammation, dyslipidemia, disturbances in erythropoiesis, iron metabolism, endothelial function, and nutritional status have been reported in these patients. Our aim was to identify any significant association of death with these disturbances, by performing a two-year follow-up study. Methods and Results. A large set of data was obtained from $189 \mathrm{HD}$ patients (55.0\% male; $66.4 \pm 13.9$ years old), including hematological data, lipid profile, iron metabolism, nutritional, inflammatory, and endothelial (dys)function markers, and dialysis adequacy. Results. 35 patients (18.5\%) died along the follow-up period. Our data showed that the type of vascular access, C-reactive protein (CRP), and triglycerides (TG) are significant predictors of death. The risk of death was higher in patients using central venous catheter $(\mathrm{CVC})$ (Hazard ratio $[\mathrm{HR}]=3.03,95 \% \mathrm{CI}=1.49-6.13$ ), with higher CRP levels (fourth quartile), compared with those with lower levels (first quartile) $(\mathrm{HR}=17.3,95 \% \mathrm{CI}=2.40-124.9)$. Patients with higher TG levels (fourth quartile) presented a lower risk of death, compared with those with the lower TG levels (first quartile) (HR $=0.18,95 \% \mathrm{CI}=0.05-0.58)$. Conclusions. The use of CVC, high CRP, and low TG values seem to be independent risk factors for mortality in HD patients.

\section{Introduction}

Patients with end-stage renal disease (ESRD) have a high mortality rate $[1,2]$ that far exceeds the mortality rate for the non-ESRD population [3]. In the past half-century, the widespread use of hemodialysis (HD) to prolong life of ESRD patients has been a remarkable achievement, preventing death from uremia in these patients. Nowadays this therapy has expanded widely and is being used by an increasing elderly patient population, leading to significant economic consequences to patients and to healthcare systems.

Our present knowledge of the mechanisms leading to increased death in this context is incomplete. In the last years, this medical field has known significant technological and pharmacological improvements. Although some evidence may suggest that mortality rate among dialysis patients has decreased over the last few years, actually, patient's survival is still low. Cardiovascular disease (CVD) has been considered 
the most common cause of death in these patients [4]. Cardiac arrest and congestive heart failure are more prominent causes of cardiovascular death than acute myocardial infarction in patients with uremia. A higher mortality rate within the first year after initiation of HD has been described (the period of highest risk for death extends to approximately 120 days after starting dialysis).

The high risk of cardiovascular morbidity and mortality in ESRD patients is associated with a high prevalence of classic cardiovascular risk factors (hypertension, diabetes mellitus, dyslipidemia, smoking, and advanced age). In addition, several uremia-related factors may also play an important role, namely, the presence of multiple comorbid conditions, fluid overload, hyperphosphoremia, high calcium-phosphorous product, anemia, left ventricular hypertrophy, inflammation, oxidative stress, endothelial dysfunction, insulin resistance, excess sympathetic tone, hyper-homocysteinemia, high levels of lipoprotein(a), and increased asymmetrical dimethylarginine [5-8]. Recent reports have highlighted the importance of noncardiovascular mortality in HD patients and that it has been underestimated [3].

Systemic inflammation is frequently present in ESRD patients, and the use of central venous catheter (CVC) has been associated with an enhanced inflammatory state [9].

The aim of the present study was to evaluate the global mortality in Portuguese ESRD patients under HD, by performing a follow-up study of two years, in order to identify any significant association of death with systemic parameters, including dialysis adequacy, nutritional status, hematological data, lipid profile, iron metabolism, and inflammatory and endothelial (dys)function markers, as well as the type of vascular access, presence of comorbidities, and with associated therapies. The parameters associated with mortality in this context may provide biomarkers to be used in the clinical setting.

\section{Methods}

2.1. Patients. This research protocol was approved by the Ethics Committee of Fresenius Medical Care, Portugal. All participants gave their written informed consent to participate in this study.

In this two-year follow-up study, starting from April 2009, 189 HD patients, from 3 dialysis clinics in the Northern region of Portugal, were included in the study. These patients were under $\mathrm{HD}$ for at least 90 days. At the start of the study, the patients were clinically evaluated and blood was collected for the analytical studies; afterwards, a clinical followup was performed during two years, in order to identify the cases of death, transplant, and transfer to peritoneal dialysis. Data regarding demographic characteristics, chronic kidney disease and medical history, dialysis, and medical prescriptions, as well as laboratory data, was also collected at the start of the study.

Diabetes was defined by the current guidelines [10] or by the use of insulin or oral hypoglycemic agents. Hypertension was defined by the current guidelines (blood pressure $>130 / 85 \mathrm{~mm} \mathrm{Hg}$ ) [11] or by the use of antihypertensive medication. Therapy with recombinant human erythropoietin (rhEPO) and with intravenous iron was based on the current guidelines. The classification of ESRD patients as responders or nonresponders to rhEPO therapy was performed in accordance with the European Best Practice Guidelines [12]. Patients with autoimmune disease, malignancy, and acute or chronic infection were excluded.

A group of 25 healthy volunteers was selected as control, based on normal hematological and biochemical values, and no history of kidney or inflammatory diseases, in order to better define the changes occurring in HD patients. This group was matched, as far as possible, for age and gender with HD patients.

2.2. Assays. Blood samples were obtained immediately before the HD procedure, in the midweek dialysis day, and processed within 2 hours after collection. Blood was collected to tubes with (EDTA) and without anticoagulant, in order to obtain whole blood, plasma, buffy-coat, and serum. Aliquots were immediately stored at $-80^{\circ} \mathrm{C}$, whenever necessary, until assayed.

Erythrocyte count, hematocrit, hemoglobin concentration, and hematimetric indices (mean cell volume (MCV), mean cell hemoglobin $(\mathrm{MCH})$, and mean cell hemoglobin concentration (MCHC)) were measured by using an automatic blood cell counter (Sysmex K1000; Sysmex, Hamburg, Germany). Leukocyte differential counts were evaluated in Wright-stained blood films. Reticulocyte count was made by microscopic counting on blood smears after vital staining with new methylene blue (reticulocyte stain; Sigma, St. Louis, MO, USA). The reticulocyte production index (RPI) was calculated, as an appropriate way to measure the effective erythrocyte production, by correcting for both changes in hematocrit (degree of anemia) and for premature reticulocyte release from the bone marrow.

Serum albumin levels were measured using a colorimetric assay end-point method (Albumin Plus; Roche $\mathrm{GmbH}$, Mannheim, Germany). Serum iron concentration was determined using a colorimetric method (Iron, Randox Laboratories Ltd., North Ireland, UK), whereas serum ferritin and serum transferrin were measured by immunoturbidimetry (Ferritin, Laboratories Ltd., North Ireland, UK; Transferrin, Laboratories Ltd., North Ireland, UK). Enzymelinked immunosorbent assays were used for measurement of plasma soluble transferrin receptors (s-TfR) (Human sTfR immunoassay, R\&D systems, MN, USA); transferrin saturation (TS) was calculated by the formula: TS (\%) = $70.9 \times$ serum iron concentration $(\mathrm{mg} / \mathrm{dL}) /$ serum transferrin concentration $(\mathrm{mg} / \mathrm{dL})$.

Plasma concentration of adiponectin, interleukin (IL)6, tissue plasminogen activator (tPA), and plasminogen activator inhibitor-1 (PAI-1) were evaluated by using standard commercial enzyme-linked immunoassays (adiponectin, IL6 ELISA High-Sensitivity, tPA, and PAI-1, all from eBioscience). D-dimer and C-reactive protein (CRP) were evaluated by immunoturbidimetry, using commercially available kits (BCS XP system, Siemens, Germany; CRP (latex) HighSensitivity, Roche Diagnostics, resp.). 
The activity of paraoxonase 1 (PON1) was assessed spectrophotometrically and expressed in nmol of p-nitrofenol/ $\mathrm{mL} / \mathrm{min}$. Briefly, PON1 activity was measured by adding serum to $1 \mathrm{~mL}$ Tris/ $\mathrm{HCl}$ buffer $(100 \mathrm{mmol} / \mathrm{L}, \mathrm{pH}$ 8.0) containing $2 \mathrm{mmol} / \mathrm{L} \mathrm{CaCl}_{2}$ and $5.5 \mathrm{mmol} / \mathrm{L}$ paraoxon (O,O-diethylO-p-nitrophenylphosphate, Sigma Chemical Co.). The rate of generation of $\mathrm{p}$-nitrophenol was determined by reading the absorbance at $412 \mathrm{~nm}, 37^{\circ} \mathrm{C}$, with the use of a continuously recording spectrophotometer (Beckman DU-68).

The evaluation of serum hepcidin concentration was performed by using an enzymatic immunoassay (Hepcidin25, EIA Kit Extraction-Free, Peninsula Laboratories, LLC, San Carlos, CA, USA).

Serum lipids, lipoproteins, and apolipoprotein analysis were performed in an autoanalyser (Cobas Mira S, Roche, Basel, Switzerland) using commercially available kits.

Serum total cholesterol and triglycerides concentrations were performed by enzymatic colorimetric tests (cholesterol oxidase-phenol aminophenazone and glycerol-3-phospate oxidase-phenol aminophenazone methods, Roche, resp.). High-density lipoprotein cholesterol (HDLc) and lowdensity lipoprotein cholesterol (LDLc) levels were measured using enzymatic colorimetric tests, after selective separation of HDL and LDL fractions (Direct HDLCholesterol and Direct LDLCholesterol, Roche). Serum levels of apolipoprotein (Apo) A-I and Apo B were evaluated by immunoturbidimetric assays (uni-kit apolipoproteinA-I and B specific antiserums, Roche). Serum Lp(a) was quantified by using an immunoturbidimetric method ( $\mathrm{Lp}(\mathrm{a})$, Roche Diagnostics). Oxidized LDL (ox-LDL) was measured directly in plasma by using a two-site enzyme immunoassay (oxidized LDL ELISA, Mercodia, Uppsala, Sweden).

2.3. Statistical Analysis. Kolmogorov-Smirnov test was used to test for normality of the variable distributions. Patients were categorized according to their status at the end of the 2 years of followup: alive (group 1) or dead (group 2). Differences between groups were analyzed by using Student's t-test or Mann-Whitney test, according to the results obtained in the Kolmogorov-Smirnov test. Normally distributed variables are presented as mean \pm SD and those nonnormally distributed are presented as median (interquartile range). Proportions were compared between groups using chi-square tests.

Survival analysis taking competing risks into account was performed to analyze patient's survival. The event of interest was death and the competing risk event was renal transplantation. Patients transferred to peritoneal dialysis were excluded (given the small number involved $(n=2)$ ). Estimates of cumulative incidence function were calculated. Regression models taking competing risks into account (Fine and Gray model based on subdistribution hazard model) were carried out, to analyze the effect of covariates in patient's survival. To decide which variables should be included in the final multivariable model, an exploratory analysis was performed by fitting models for each variable in turn, adjusting for age and previous time in HD. The final multivariable model included all of these candidate variables with $P$ values $<0.05$. All analyses were performed with SPSS and R software using the packages cmprsk, and significance level $(\alpha)$ was set at 0.05 .

\section{Results}

3.1. Patient's Demographic and Clinical Data. We studied 189 ESRD patients under HD (104 male, 85 female; mean age: 66.4 years, SD 13.9 years, range $27.6-92.0$ years). The patients were under therapeutic HD three times per week, for 3-5 hours, and were under HD for 2.13 (0.82-5.28) years, when they were included in this study. All patients used the high-flux polysulfone FX-class dialyzers $\left(1.4-2.2 \mathrm{~m}^{2}\right)$ of Fresenius (Bad Hamburg, Germany). Arteriovenous fistula (AVF) was used by 146 patients $(77.2 \%)$, whereas 43 patients $(22.8 \%)$ had a CVC. The main causes of renal failure in these patients were diabetes mellitus $(n=68)$, followed by arterial hypertension $(n=25)$, glomerulonephritis $(n=16)$, polycystic renal disease $(n=12)$, reflux nephropathy $(n=$ $8)$, obstructive uropathy $(n=4)$, hereditary nephropathy $(n=3)$, chronic interstitial nephritis $(n=2)$, nephrolithiasis $(n=3)$, other diseases $(n=7)$, and uncertain etiology $(n=40)$. Sixteen $(8.5 \%)$ patients were classified as resistant to rhEPO therapy. A total number of 108 patients $(63.9 \%)$ had arterial hypertension, 103 patients (54.5\%) were under antihypertensive treatment, 75 (39.7\%) were using statins, 127 $(67.2 \%)$ were under intravenous iron therapy, 71 (39.0\%) were under anti platelet therapy, and $8(4.6 \%)$ were under oral hypocoagulation.

3.2. Analytical Data. The analytical results for the patients at starting of the follow-up study, and for the control group, are presented in Table 1. The hematological study showed that the HD patients experience a significant decrease in hemoglobin concentration, hematocrit, erythrocyte, platelets and lymphocyte counts, and a significant increase in MCV, as compared with the control; several significant changes were also observed in iron metabolism (an increase in transferrin saturation, sTfR, ferritin and hepcidin, and a decrease in iron and transferrin), in inflammatory markers (increase in adiponectin, IL-6, and CRP and a decrease in PON1), in the lipid profile (increase in oxLDL/LDL ratio and a decrease in total cholesterol, HDLc, LDLc, Apo A, Apo B and oxLDL), in endothelial (dys)function markers (an increase in tPA, tPA/PAI-1 ratio and D-dimers, and a decrease in PAI-1); a significant increase in BMI, was also observed in patients, when compared with the control group.

3.3. Outcome. During the follow-up period (24 months), 35 patients died (18.5\%) and 18 patients received a kidney transplant. Table 2 shows the values of the parameters that presented significant differences, when considering dead and alive patients at the end of the 2-year follow-up period. Statistically significant differences were found, concerning dialysis markers, nutritional status, hematological data, iron metabolism, inflammation, lipid profile, and endothelial function. The patients that died during the study period showed significantly lower values for calcium, calcium phosphorus product, creatinine, ultrafiltration volume, transferrin, transferrin saturation, triglycerides, VLDL, Apo A, BMI, 
TABLE 1: Baseline values of the 189 ESRD patients under HD therapy.

\begin{tabular}{|c|c|c|}
\hline & Controls $(n=25)$ & HD patients $(n=189)$ \\
\hline \multicolumn{3}{|c|}{ Biochemical and dialysis markers } \\
\hline URR, \% & - & $75.9 \pm 6.56$ \\
\hline $\mathrm{KT} / \mathrm{Ve}$ & - & $1.48 \pm 0.31$ \\
\hline Ultrafiltration volume, $\mathrm{L}$ & - & $2.43 \pm 0.94$ \\
\hline Creatinine, $\mathrm{mg} / \mathrm{dL}$ & - & $8.14 \pm 2.81$ \\
\hline Potassium, mmol/L & - & $5.40(4.80-5.88)$ \\
\hline Sodium, $\mathrm{mmol} / \mathrm{L}$ & - & $137(135-139)$ \\
\hline Phosphorus, $\mathrm{mmol} / \mathrm{L}$ & - & $4.48(3.60-5.40)$ \\
\hline Calcium, mg/dL & - & $8.60(8.10-9.10)$ \\
\hline Calcium phosphorus product & - & $37.58(31.15-44.86)$ \\
\hline Darbopoeitin, $\mu \mathrm{g} / \mathrm{kg} /$ week & - & $0.42(0.20-0.75)$ \\
\hline \multicolumn{3}{|c|}{ Hematological data } \\
\hline Erythrocytes, $\times 10^{12} / \mathrm{L}$ & $4.9 \pm 0.45$ & $3.8 \pm 0.53^{*}$ \\
\hline Hemoglobin, g/dL & $14.9 \pm 0.86$ & $11.7 \pm 1.44^{*}$ \\
\hline Hematocrit, \% & $44.3 \pm 2.80$ & $36.5 \pm 4.59^{*}$ \\
\hline $\mathrm{MCV}, \mathrm{fL}$ & $88.1 \pm 11.72$ & $95.8 \pm 6.01^{*}$ \\
\hline $\mathrm{MCHC}, \mathrm{g} / \mathrm{dL}$ & $33.8 \pm 0.46$ & $32.3 \pm 1.19$ \\
\hline RDW, \% & $15.1 \pm 0.54$ & $15.1 \pm 1.91$ \\
\hline Platelets, $\times 10^{9} / \mathrm{L}$ & $257.1 \pm 58.85$ & $184.1 \pm 55.4^{*}$ \\
\hline Leukocytes, $\times 10^{9} / \mathrm{L}$ & $6.7 \pm 1.66$ & $6.4 \pm 2.0$ \\
\hline Neutrophils, $\times 10^{9} / \mathrm{L}$ & $4.3 \pm 1.48$ & $4.0 \pm 1.49$ \\
\hline Lymphocytes, $\times 10^{9} / \mathrm{L}$ & $2.0 \pm 0.37$ & $1.7 \pm 0.70^{*}$ \\
\hline Neutrophil/lymphocyte ratio & $2.2 \pm 0.78$ & $2.7 \pm 1.51$ \\
\hline \multicolumn{3}{|c|}{ Iron metabolism markers } \\
\hline Transferrin, mg/dL & $310.8 \pm 44.04$ & $184.4 \pm 35.8^{*}$ \\
\hline Transferrin saturation, $\%$ & $12.7 \pm 4.73$ & $15.1(11.8-19.3)^{*}$ \\
\hline Iron, $\mu \mathrm{g} / \mathrm{dL}$ & $55.0 \pm 17.97$ & $38.0(30.0-53.5)^{*}$ \\
\hline sTfR, nmol/L & $13.6 \pm 3.72$ & $23.3 \pm 11.9^{*}$ \\
\hline Ferritin, ng/mL & $102.5 \pm 60.61$ & $400.8 \pm 152.9^{*}$ \\
\hline Hepcidin-25, ng/mL & $218.4(124.5-318.6)$ & $1599.1(863.6-2409.0)^{*}$ \\
\hline \multicolumn{3}{|c|}{ Inflammatory markers } \\
\hline IL-6, pg/mL & $0.43(0.28-0.61)$ & $2.29(1.40-4.26)^{*}$ \\
\hline $\mathrm{CRP}, \mathrm{mg} / \mathrm{dL}$ & $0.75(0.44-1.91)$ & $5.13(2.33-13.05)^{*}$ \\
\hline PON1, nmol p-nitrofenol $/ \mathrm{mL} / \mathrm{min}$ & $466.8(383.8-535.8)$ & $361.1(323.3-463.7)^{*}$ \\
\hline Adiponectin, mg/L & $4.93 \pm 2.30$ & $9.27 \pm 4.71^{*}$ \\
\hline \multicolumn{3}{|c|}{ Lipid profile } \\
\hline Total cholesterol, mg/dL & $202.2 \pm 26.25$ & $154.2 \pm 43.5^{*}$ \\
\hline Triglycerides, mg/dL & $103.0(86.5-154.0)$ & $117.0(90.5-176.5)$ \\
\hline $\mathrm{HDLc}, \mathrm{mg} / \mathrm{dL}$ & $48.7 \pm 12.94$ & $42.2 \pm 13.5^{*}$ \\
\hline LDLc, mg/dL & $105.9 \pm 25.29$ & $73.4 \pm 29.3^{*}$ \\
\hline VLDL, mg/dL & $20.6(17.3-30.8)$ & $23.4(18.0-35.4)$ \\
\hline $\mathrm{Lp}(\mathrm{a}), \mathrm{mg} / \mathrm{dL}$ & $27.7(17.3-69.9)$ & $45.4(25.6-89.0)$ \\
\hline Apo Al, mg/dL & $145.2 \pm 26.91$ & $122.7 \pm 30.4^{*}$ \\
\hline Apo B, mg/dL & $91.4 \pm 14.49$ & $72.4 \pm 21.7^{*}$ \\
\hline Ox-LDL, U/L & $40.8 \pm 10.97$ & $33.7(27.7-40.5)^{*}$ \\
\hline OxLDL/LDL ratio, U/mg & $0.040 \pm 0.013$ & $0.052 \pm 0.018^{*}$ \\
\hline
\end{tabular}


TABle 1: Continued.

\begin{tabular}{|c|c|c|}
\hline & Controls $(n=25)$ & HD patients $(n=189)$ \\
\hline \multicolumn{3}{|c|}{ Endotelial (dys) function markers } \\
\hline $\mathrm{tPA}, \mathrm{ng} / \mathrm{mL}$ & $3.93(2.95-5.93)$ & $5.35(3.72-8.18)^{*}$ \\
\hline PAI-1, ng/mL & $31.0(15.3-59.2)$ & $18.7(12.1-32.4)^{*}$ \\
\hline tPA/PAI-1 ratio & $0.16(0.09-0.20)$ & $0.29(0.18-0.39)^{*}$ \\
\hline D-dimers, $\mathrm{ng} / \mathrm{mL}$ & $0.25(0.18-0.34)$ & $0.71(0.38-1.56)^{*}$ \\
\hline \multicolumn{3}{|c|}{ Nutritional markers } \\
\hline BMI, $\mathrm{Kg} / \mathrm{m}^{2}$ & $22.2 \pm 2.26$ & $25.9 \pm 4.63^{*}$ \\
\hline Albumin, g/dL & - & $3.9 \pm 0.38$ \\
\hline
\end{tabular}

TABLE 2: Baseline values in ESRD patients under HD therapy for variables in which significant differences were found when the patients were divided in two groups (alive or dead 2 years later).

\begin{tabular}{|c|c|c|c|}
\hline & Alive at followup (135 patients) & Dead during followup (35 patients) & $P$ \\
\hline \multicolumn{4}{|c|}{ Biochemical and dialysis markers } \\
\hline Calcium, mg/dL & $8.70(8.20-9.10)$ & $8.40(8.00-8.90)$ & 0.046 \\
\hline Calcium phosphorus product & $38.6(31.4-46.5)$ & $35.8(29.2-39.2)$ & 0.025 \\
\hline Creatinine, $\mathrm{mg} / \mathrm{dL}$ & $8.63 \pm 2.86$ & $6.45 \pm 2.10$ & $<0.001$ \\
\hline Ultrafiltration volume, $\mathrm{L}$ & $2.47 \pm 0.85$ & $2.29 \pm 1.22$ & 0.037 \\
\hline Darbopoeitin- $\alpha, \mu \mathrm{g} / \mathrm{kg} /$ week & $0.40(0.77-0.66)$ & $0.87(0.36-1.47)$ & $<0.001$ \\
\hline \multicolumn{4}{|c|}{ Hematological data } \\
\hline RDW, \% & $14.5 \pm 1.43$ & $16.9 \pm 2.32$ & $<0.001$ \\
\hline \multicolumn{4}{|c|}{ Iron metabolism markers } \\
\hline Transferrin, mg/dL & $187.6 \pm 34.1$ & $172.5 \pm 41.6$ & 0.050 \\
\hline Transferrin saturation, $\%$ & $15.8(12.3-19.7)$ & $13.5(9.67-19.1)$ & 0.032 \\
\hline \multicolumn{4}{|c|}{ Inflammatory markers } \\
\hline IL-6, pg/mL & $2.21(1.37-3.60)$ & $4.27(2.10-6.57)$ & $<0.001$ \\
\hline $\mathrm{CRP}, \mathrm{mg} / \mathrm{dL}$ & $3.93(2.09-9.73)$ & $14.9(5.15-30.4)$ & $<0.001$ \\
\hline \multicolumn{4}{|c|}{ Lipid profile } \\
\hline Triglyceride, mg/dL & $120.0(95.0-180.0)$ & $103.0(82.0-125.0)$ & 0.015 \\
\hline $\mathrm{VLDL}, \mathrm{mg} / \mathrm{dL}$ & $24.0(18.8-36.0)$ & $20.6(16.4-25.0)$ & 0.021 \\
\hline Apo A1, mg/dL & $124.3 \pm 29.7$ & $109.4 \pm 26.2$ & 0.014 \\
\hline \multicolumn{4}{|c|}{ Endotelial (dys) function markers } \\
\hline PAI-1, ng/mL & $17.9(12.3-30.4)$ & $27.4(14.2-46.9)$ & 0.034 \\
\hline D-dimers, ng/mL & $0.70(0.40-1.29)$ & $1.47(0.63-2.28)$ & 0.005 \\
\hline \multicolumn{4}{|c|}{ Nutritional markers } \\
\hline Body mass index, $\mathrm{kg} / \mathrm{m}^{2}$ & $25.4(23.0-28.5)$ & $23.3(20.5-27.3)$ & 0.008 \\
\hline Albumin, g/dL & $4.00(3.78-4.20)$ & $3.70(3.37-3.94)$ & $<0.001$ \\
\hline
\end{tabular}

Data are presented as means and standard deviation or median (interquartile range). RDW: red blood cells distribution width; IL-6: interleukin-6; CRP: Creactive protein; VLDL: very low-density lipoprotein; Apo A: apolipoprotein A; PAI-1: plasminogen activator inhibitor 1.

and albumin; significantly higher values were found for RDW, IL-6, CRP, PAI-1, and tPA. Moreover, dead patients required significantly higher rhEPO doses to achieve the target hemoglobin levels and presented a significantly higher percentage of patients using CVC (38\% versus $15 \%, P<$ 0.001 ) as the vascular access for the HD procedure.

Figure 1 summarizes the cumulative incidence curves estimating all possible outcomes, taking competing risks into account. The probability of death by $6,12,18$, and 24 months after starting the study was $0.053,0.106,0.154$, and 0.176 , respectively.

Survival regression models, all adjusted for age and previous time in HD, are presented in Table 3. This analysis showed that the type of vascular access (the use of CVC), CRP, and tryglicerides are significant predictors of the event of interest, death. Indeed, the risk of death was higher for 
TABLE 3: Survival regression models for ESRD patient's mortality.

\begin{tabular}{|c|c|c|c|c|c|c|}
\hline & \multicolumn{3}{|c|}{ Model $1^{*}$} & \multicolumn{3}{|c|}{ Final model ${ }^{* *}$} \\
\hline & Adjusted hazard ratio & $95 \% \mathrm{CI}$ & $P$ & Adjusted hazard ratio & $95 \% \mathrm{CI}$ & $P$ \\
\hline \multicolumn{7}{|c|}{ Type of vascular access } \\
\hline CVC versus AVF & 2.65 & $1.33-5.30$ & 0.006 & 3.03 & $1.49-6.13$ & 0.002 \\
\hline \multicolumn{7}{|l|}{ C-reactive protein } \\
\hline Q2 versus Q1 & 6.01 & $0.79-46.7$ & 0.083 & 4.91 & $0.62-38.6$ & 0.130 \\
\hline Q3 versus Q1 & 8.04 & $1.04-62.2$ & 0.046 & 8.31 & $1.09-63.1$ & 0.041 \\
\hline Q4 versus Q1 & 18.1 & $2.51-130.0$ & 0.004 & 17.3 & $2.40-124.9$ & 0.005 \\
\hline \multicolumn{7}{|l|}{ Triglycerides } \\
\hline Q2 versus Q1 & 0.46 & $0.20-1.02$ & 0.057 & 0.48 & $0.19-1.21$ & 0.120 \\
\hline Q3 versus Q1 & 0.48 & $0.20-1.13$ & 0.093 & 0.48 & $0.17-1.34$ & 0.160 \\
\hline Q4 versus Q1 & 0.22 & $0.07-0.75$ & 0.015 & 0.18 & $0.05-0.58$ & 0.004 \\
\hline
\end{tabular}

* Model 1: hazard ratio adjusted for age and previous time on $\mathrm{HD} ;{ }^{* *}$ Final model: hazard ratio adjusted for all variable in the model. CVC: central venous catheter; AVF: arteriovenous fistula; Q: quartile; CI: confidence interval.

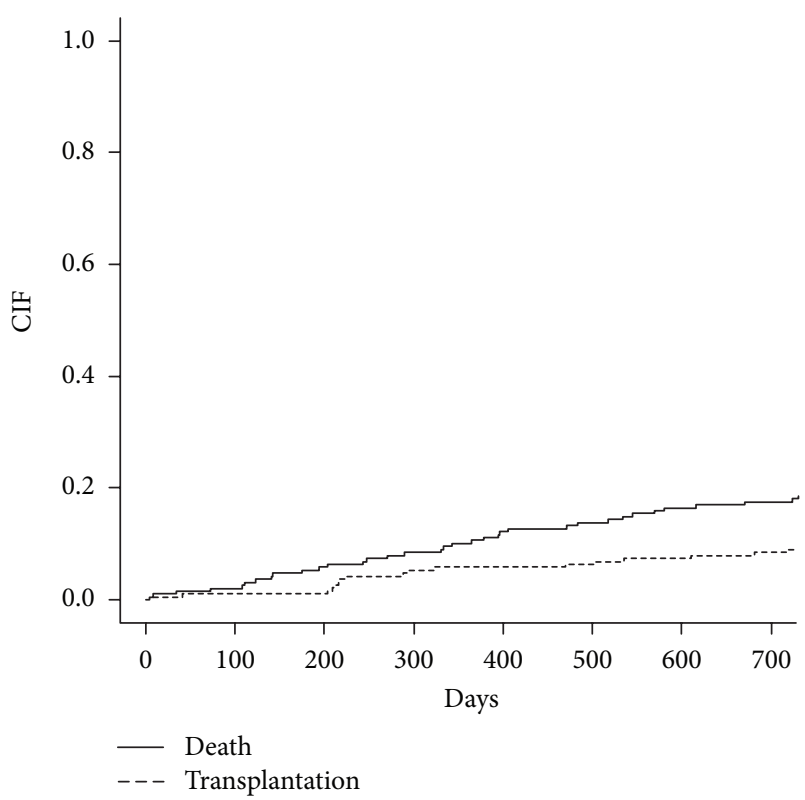

FIGURE 1: Cumulative incidence curves for all possible events (death as event of interest and transplantation as competing risk) for ESRD patients.

CVC (hazard ratio $(\mathrm{HR})=3.03,95 \% \mathrm{CI}=1.49-6.13$ ) and for patients with higher levels of CRP (4th quartile), as compared with the patients with lower level (1st quartile) ( $\mathrm{HR}=17.3,95 \%$ $\mathrm{CI}=2.40-124.9$ ) (Table 3). Patients with tryglicerides levels in the 4th quartile had a lower risk of death, as compared with those patients in the 1st quartile ( $\mathrm{HR}=0.18,95 \% \mathrm{CI}=0.05$ $0.58)$.

\section{Discussion}

In the present study, 189 ESRD patients under HD therapy were studied at the start of the study and followed for 2 years, in order to identify parameters that could be associated with mortality and, therefore, to provide biomarkers of risk.

The mortality rate in HD remains high $[3,13]$, in spite of the considerable improvements in medical and technical support to these patients in the last years. CVD events are the main causes of mortality in ESRD patients [4]. However, it has been argued that noncardiovascular causes of death seem to be increasing to the same extent as cardiovascular mortality in ESRD patients [3]. Shastri et al. studied the patterns of death in a large cohort of HD patients and showed that sudden cardiac death accounted for $22 \%$ of the fatal events, whereas nonsudden cardiac death and noncardiac death accounted for $17 \%$ and $61 \%$, respectively [14].

In our study, during the follow-up period, 35 patients died $(18.5 \%)$ and 18 patients received a kidney transplant. Considering dead and living patients at the end of the 2year follow-up period, we found that several variables were significantly different for the two groups of patients. We found that the patients who died during the follow period of the study, as compared to living patients, had lower HD efficacy, as showed by lower values of calcium, calcium phosphorus product, creatinine, and ultrafiltration volume; higher disturbances in iron metabolism and erythropoiesis, as shown by lower values of transferrin and transferrin saturation and higher values of RDW; a poor nutritional status, as reflected by the lower values of triglycerides, VLDL, Apo A, BMI, and albumin; a higher inflammatory condition, as shown by the higher values of IL- 6 and CRP; endothelial and hemostatic disturbances, as suggested by the higher values of PAI-1 and D-dimers; and the development of resistance to rhEPO therapy, as they needed higher rhEPO doses to achieve hemoglobin concentrations within the target range; a higher percentage of them used CVC for vascular access. After performing a multivariate analysis, we observed that the use of CVC as the vascular access for the HD procedure, high CRP levels, and low triglycerides levels were independently associated with mortality.

The present work, showing an association between mortality in ESRD patients under HD treatment and both the type of vascular access (CVC) and CRP, shows that inflammation plays an important role in the mortality of these patients [15] and that their values can be used as predictors of death. Whether inflammation has any significant role in the physiopathology of ESRD, leading to death, or is merely a risk marker remains to be established. Zimmermann et al., 
studying $280 \mathrm{HD}$ patients, showed CRP as an independent predictor of both overall death and cardiovascular death [15], which is in agreement with our findings.

In a previous report [9], we showed that ESRD patients with a CVC had lower levels of hemoglobin and albumin and higher levels of CRP, IL-6, D-dimers, and tPA, when compared to ESRD patients using AVF [5]. This association between CVC use and the enhancement of the inflammatory process and endothelial dysfunction could justify the high mortality rate found in ESRD patients using CVC. Several other reports $[16,17]$ have shown an association between the type of vascular access and mortality in HD patients.

Low values for plasma triglycerides were also associated with mortality in this study. This finding must be seen in the context of a significant importance of malnutrition in this type of patients [18]. Owen et al. showed serum albumin concentration as a powerful predictor of death in a cohort of 13, $473 \mathrm{HD}$ patients [18]. In our study, albumin presented lower values in patients that died during followup; however, the survival regression models did not show albumin as an independent risk factor. The low levels of triglycerides might reflect a "malnutrition-inflammation complex syndrome" [19] or, more probably, a hemodialysis-catabolic state, associated with increased mortality.

Most of the changes (Table 1) observed in the characterization of $\mathrm{HD}$ patients are in accordance with the literature. HD patients presented with anemia, as shown by a decreased hemoglobin concentration, hematocrit, and erythrocyte count. They also presented a decrease in platelet and lymphocyte counts. The lymphocytopenia seems to result, at least in part, from a decrease in total circulating CD3+ T-lymphocytes and affects both the CD4+ and the CD8+ T-cell subsets [20]. The presence of inflammatory features (increased levels of IL- 6 and CRP and decreased PON1 activity) were consistent with findings reported in HD patients [21-23]; however, the causes for the inflammatory response are not well clarified. Indeed, there are several potential sources, including bacterial contamination of the dialyser, incompatibility with the dialyser membrane, and the vascular access. It is known that some inflammatory players, for example, hepcidin, have an important impact in iron metabolism, reducing the mobilization and absorption of iron for erythropoiesis [24], leading, therefore, to an iron depleted erythropoiesis. In accordance with this, we found an increase in sTfR, ferritin, and transferrin saturation and a decrease in transferrin and in iron serum levels. IL-6, which is significantly increased in HD patients (Table 1), is known to strongly induce hepcidin mRNA expression [25, 26]. Thus, the high levels of hepcidin found in HD patient are explained by the underlying chronic inflammation. Recent studies have reported that higher body mass index (BMI) plays a protective role in HD patients [27-29]. Actually, the increased $\mathrm{BMI}$ in our $\mathrm{HD}$ patients was associated with higher adiponectin serum levels. Adiponectin is an adipokine that exerts a variety of anti-inflammatory activities, interfering with macrophage function by inhibiting phagocytosis, the production of IL- 6 and TNF $\alpha$, T-cell function, and by promoting the release of IL-10 and IL-1 receptor antagonists $[30,31]$. The increase in adiponectin suggests that the adipose tissue in HD patients may influence or modulate the inflammatory response. Several changes in the lipid profile were also found, namely, a decrease in total cholesterol, HDLc, LDLc, ApoA, ApoB, and Ox-LDL. In spite of the decrease in OxLDL, a higher ox-LDL/LDL ratio was found, showing a higher proportion of oxidized LDL particles in HD patients. It is known that ox-LDLs present important proinflammatory and procoagulant properties [32], and, therefore, it may further contribute to inflammatory changes. Endothelial function was also disturbed, as shown by the increase in D-dimers and tPA/PAI-1 ratio. It is uncertain if the higher values of fibrin fragment $\mathrm{D}$-dimers in $\mathrm{HD}$ patients result from a higher activation of coagulation and/or of fibrinolysis, though the higher tPA values and tPA/PAI-1 ratio suggest an increase in fibrinolysis and a disturbance in endothelial function.

This study presented some limitations, namely, the number of patients included in this study and the follow-up period that were relatively limited.

In conclusion, our study showed that the type of vascular access for HD procedure, inflammation, and the nutritional status of the patients are important factors for the survival of these patients and that the use of CVC, CRP, and TG values may provide good biomarkers of risk in ESRD patients under HD.

\section{Acknowledgments}

This study was supported by "Fundação para a Ciência e Tecnologia" (FCT: PIC/IC/83221/2007) and cofinanced by FEDER (FCOMP-01-0124-FEDER-008468).

\section{References}

[1] W. M. Vollmer, P. W. Wahl, and C. R. Blagg, "Survival with dialysis and transplantation in patients with end-stage renal disease," The New England Journal of Medicine, vol. 308, no. 26, pp. 1553-1558, 1983.

[2] J. P. Held, F. Brunner, M. Odaka, J. R. Garcia, F. K. Port, and D. S. Gaylin, "Five-year survival for end-stage renal disease patients in the United States, Europe, and Japan, 1982 to 1987," American Journal of Kidney Diseases, vol. 15, no. 5, pp. 451-457, 1990.

[3] K. J. Jager, B. Lindholm, D. Goldsmith et al., "Cardiovascular and non-cardiovascular mortality in dialysis patients: where is the link?" Kidney International Supplements, vol. 1, pp. 21-23, 2011.

[4] R. N. Foley, P. S. Parfrey, and M. J. Sarnak, "Clinical epidemiology of cardiovascular disease in chronic renal disease," American Journal of Kidney Diseases, vol. 32, no. 5, supplement 3, pp. S112-S119, 1998.

[5] M. J. Sarnak, A. S. Levey, A. C. Schoolwerth et al., "Kidney disease as a risk factor for development of cardiovascular disease: a statement from the American Heart Association Councils on Kidney in Cardiovascular Disease, High Blood Pressure Research, Clinical Cardiology, and Epidemiology and Prevention," Circulation, vol. 108, no. 17, pp. 2154-2169, 2003.

[6] A. J. Collins, "Cardiovascular mortality in end-stage renal disease," American Journal of the Medical Sciences, vol. 325, no. 4, pp. 163-167, 2003. 
[7] P. Stenvinkel, J. J. Carrero, J. Axelsson, B. Lindholm, O. Heimbürger, and Z. Massy, "Emerging biomarkers for evaluating cardiovascular risk in the chronic kidney disease patient: how do new pieces fit into the uremic puzzle?" Clinical Journal of the American Society of Nephrology, vol. 3, no. 2, pp. 505-521, 2008.

[8] J. Kendrick and M. B. Chonchol, "Nontraditional risk factors for cardiovascular disease in patients with chronic kidney disease," Nature Clinical Practice Nephrology, vol. 4, no. 12, pp. 672-681, 2008.

[9] E. Costa, S. Rocha, P. Rocha-Pereira et al., "Cross-talk between inflammation, coagulation/fibrinolysis and vascular access in hemodialysis patients," Journal of Vascular Access, vol. 9, no. 4, pp. 248-253, 2008.

[10] American Diabetes Association, "Standards of medical care in diabetes-2009,” Diabetes Care, vol. 32, pp. S13-S61, 2009.

[11] JNC 6. National High Blood Pressure Education Program, "The sixth report of the Joint National Committee on Prevention, Detection, Evaluation, and Treatment of High Blood Pressure," Archives of Internal Medicine, vol. 157, no. 21, pp. 2413-2446, 1997.

[12] F. Locatelli, P. Aljama, P. Bárány et al., "Revised European best practice guidelines for the management of anaemia in patients with chronic renal failure," Nephrology, Dialysis, Transplantation, vol. 19, supplement 2, pp. 1-47, 2004.

[13] B. A. Cooper, P. Branley, L. Bulfone et al., "A randomized, controlled trial of early versus late initiation of dialysis," The New England Journal of Medicine, vol. 363, no. 7, pp. 609-619, 2010.

[14] S. Shastri, N. Tangri, H. Tighiouart et al., "Predictors of sudden cardiac death: a competing risk approach in the hemodialysis study," Clinical Journal of the American Society of Nephrology, vol. 7, no. 1, pp. 123-130, 2012.

[15] J. Zimmermann, S. Herrlinger, A. Pruy, T. Metzger, and C. Wanner, "Inflammation enhances cardiovascular risk and mortality in hemodialysis patients," Kidney International, vol. 55, no. 2, pp. 648-658, 1999.

[16] R. K. Dhingra, E. W. Young, T. E. Hulbert-Shearon, S. F. Leavey, and F. K. Port, "Type of vascular access and mortality in U.S. hemodialysis patients," Kidney International, vol. 60, no. 4, pp. 1443-1451, 2001.

[17] S. Pastan, J. M. Soucie, and W. M. McClellan, "Vascular access and increased risk of death among hemodialysis patients," Kidney International, vol. 62, no. 2, pp. 620-626, 2002.

[18] W. F. Owen Jr., N. L. Lew, Y. Liu, E. G. Lowrie, and J. M. Lazarus, "The urea reduction ratio and serum albumin concentration as predictors of mortality in patients undergoing hemodialysis," The New England Journal of Medicine, vol. 329, no. 14, pp. 10011006, 1993.

[19] K. Kalantar-Zadeh, R. D. Kilpatrick, N. Kuwae et al., "Revisiting mortality predictability of serum albumin in the dialysis population: time dependency, longitudinal changes and populationattributable fraction," Nephrology Dialysis Transplantation, vol. 20, no. 9, pp. 1880-1888, 2005.

[20] E. Costa, M. Lima, J. M. Alves et al., "Inflammation, T-cell phenotype, and inflammatory cytokines in chronic kidney disease patients under hemodialysis and its relationship to resistance to recombinant human erythropoietin therapy," Journal of Clinical Immunology, vol. 28, no. 3, pp. 268-275, 2008.

[21] E. Costa, B. J. G. Pereira, P. Rocha-Pereira et al., "Role of prohepcidin, inflammatory markers and iron status in resistance to rhEPO therapy in hemodialysis patients," American Journal of Nephrology, vol. 28, no. 4, pp. 677-683, 2008.
[22] J. Bazeley, B. Bieber, Y. Li et al., "C-reactive protein and prediction of 1-year mortality in prevalent hemodialysis patients," Clinical Journal of the American Society of Nephrology, vol. 6, no. 10, pp. 2452-2461, 2011.

[23] C. Libetta, V. Sepe, P. Esposito, F. Galli, and A. Dal Canton, "Oxidative stress and inflammation: implications in uremia and hemodialysis," Clinical Biochemistry, vol. 44, no. 14-15, pp. 11891198, 2011.

[24] M. L. V. Jacober, R. L. Mamoni, C. S. P. Lima, B. L. Dos Anjos, and H. Z. W. Grotto, "Anaemia in patients with cancer: role of inflammatory activity on iron metabolism and severity of anaemia," Medical Oncology, vol. 24, no. 3, pp. 323-329, 2007.

[25] R. E. Fleming and W. S. Sly, "Hepcidin: a putative ironregulatory hormone relevant to hereditary hemochromatosis and the anemia of chronic disease," Proceedings of the National Academy of Sciences of the United States of America, vol. 98, no. 15, pp. 8160-8162, 2001.

[26] D. M. Wrighting and N. C. Andrews, "Interleukin-6 induces hepcidin expression through STAT3," Blood, vol. 108, no. 9, pp. 3204-3209, 2006.

[27] R. M. - Tanner, T. M. Brown, and P. Muntner, "Epidemiology of obesity, the metabolic syndrome, and chronic kidney disease," Current Hypertension Reports, vol. 14, pp. 152-159, 2012.

[28] K. Kalantar-Zadeh, K. C. Abbott, A. K. Salahudeen, R. D. Kilpatrick, and T. B. Horwich, "Survival advantages of obesity in dialysis patients," American Journal of Clinical Nutrition, vol. 81, no. 3, pp. 543-554, 2005.

[29] R. G. Kalaitzidis and K. C. Siamopoulos, "The role of obesity in kidney disease: recent findings and potential mechanisms," International Urology and Nephrology, vol. 43, no. 3, pp. 771784, 2011.

[30] G. Fantuzzi, "Adipose tissue, adipokines, and inflammation," Journal of Allergy and Clinical Immunology, vol. 115, no. 5, pp. 911-920, 2005.

[31] G. Fantuzzi, "Adiponectin and inflammation: consensus and controversy," Journal of Allergy and Clinical Immunology, vol. 121, no. 2, pp. 326-330, 2008.

[32] A. N. N. Mertens and P. Holvoet, "Oxidized LDL and HDL: antagonists in atherothrombosis," FASEB Journal, vol. 15, no. 12, pp. 2073-2084, 2001. 


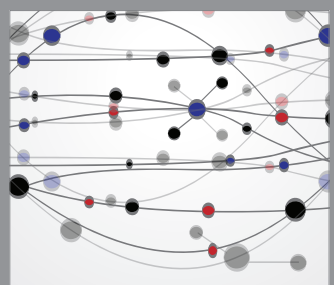

The Scientific World Journal
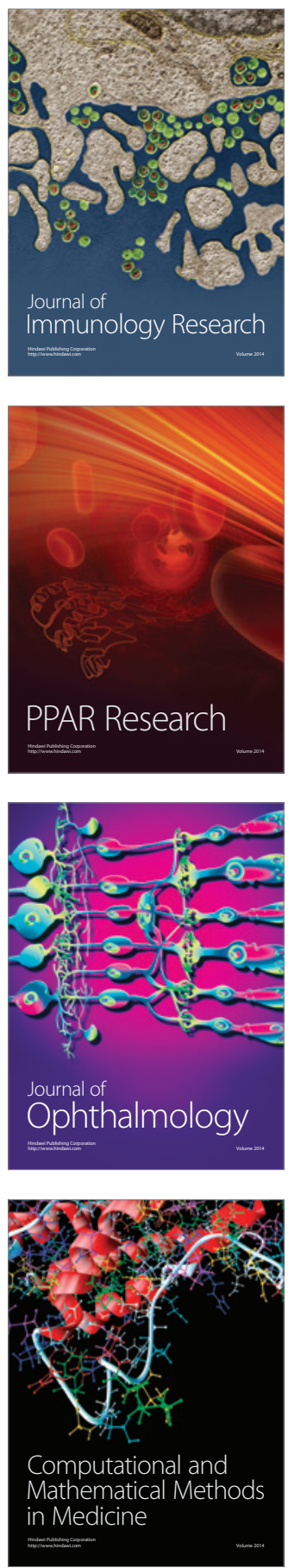

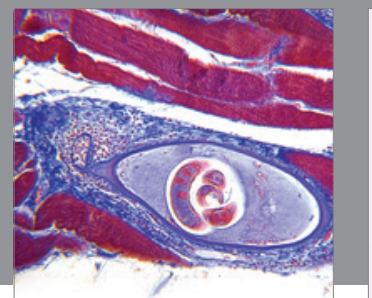

Gastroenterology

Research and Practice
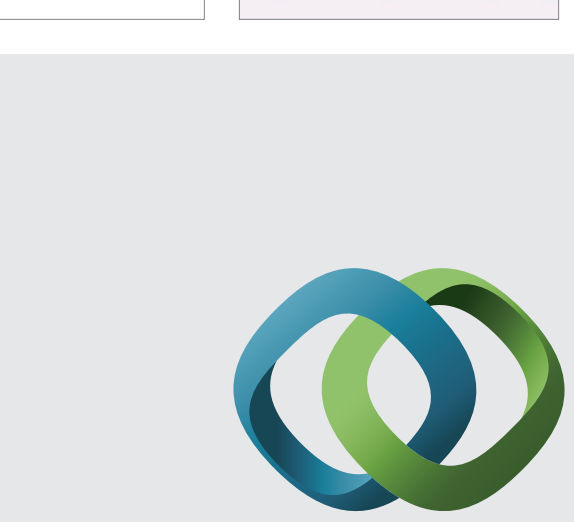

\section{Hindawi}

Submit your manuscripts at

http://www.hindawi.com
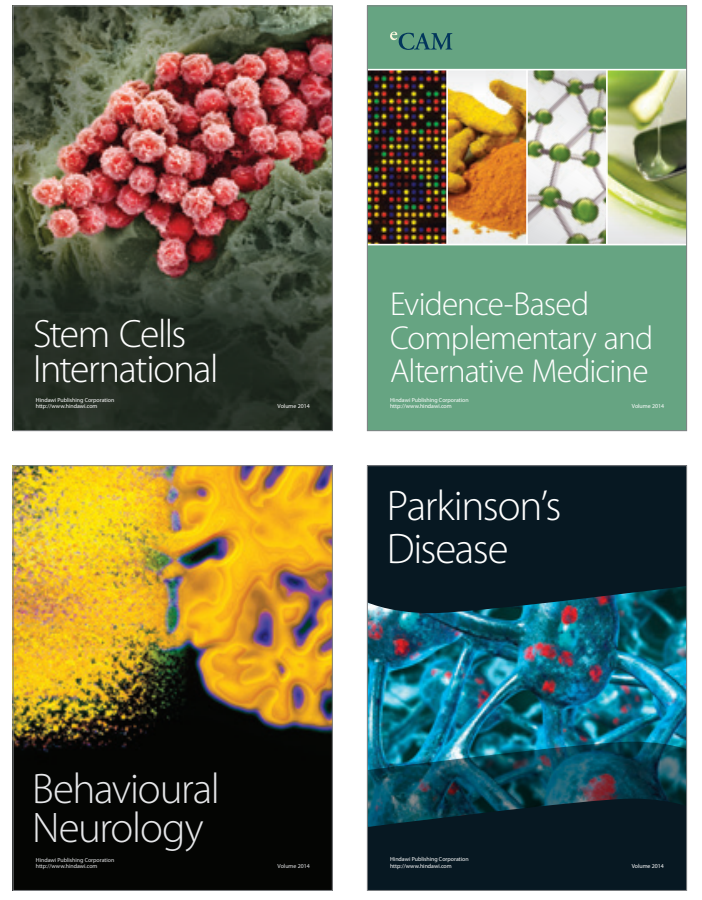
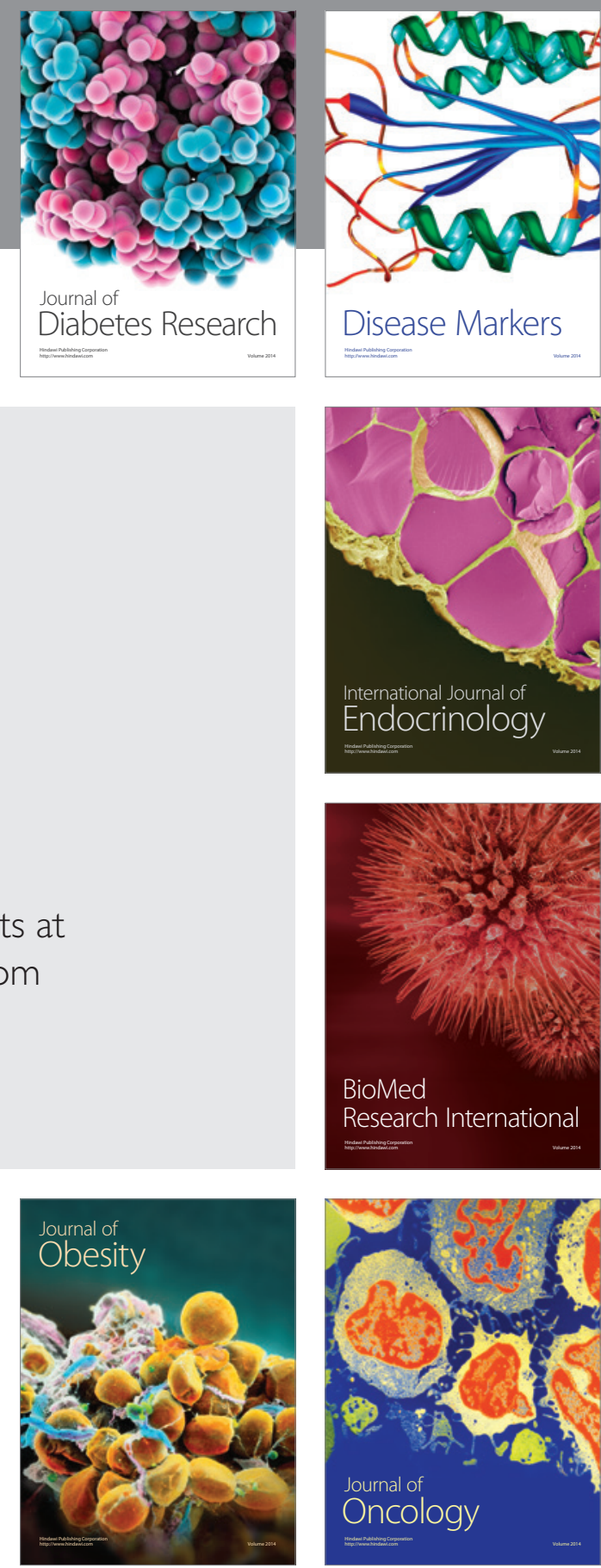

Disease Markers
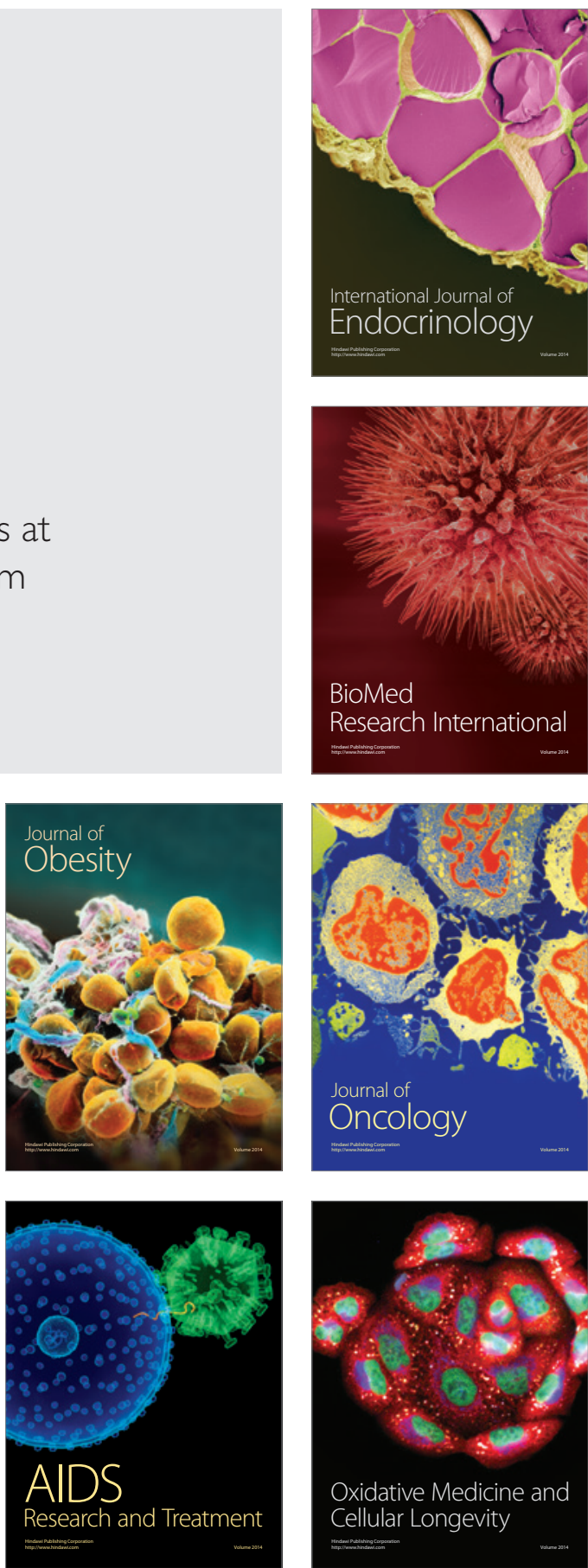